\title{
The impact of semantic distance and induced stress on analogical reasoning: A neurocomputational account
}

\author{
Michael Vendetti • Barbara J. Knowlton • \\ Keith J. Holyoak
}

Published online: 16 June 2012

(C) Psychonomic Society, Inc. 2012

\begin{abstract}
In a study of reasoning with four-term verbal analogy problems, we explored the relationship between the effects of an acute, mild stressor and the complexity of the reasoning process. Participants judged whether analogy problems in the form $A: B:: C: D$ were valid or invalid, on the basis of whether the relation in the $\mathrm{A}: \mathrm{B}$ term matched that in the $C: D$ term. Half of the problems contained a $C: D$ pair semantically near the A:B pair (e.g., NOSE:SCENT :: TONGUE:TASTE), and the other half contained ones semantically far from A:B (e.g., NOSE:SCENT :: ANTENNA:SIGNAL). After an initial block without stress, participants were randomly assigned to count backward by $13 \mathrm{~s}$ from 1,000 while being told to go faster, or to count forward by $1 \mathrm{~s}$ from 0 . The stress-induced participants reported a significant increase in state anxiety as compared to controls immediately after the mental arithmetic task. Stressed participants performed less accurately (as measured by $d^{\prime}$ ) on both near and far analogy problems, mainly due to an increase in false alarms. We were able to model the influence of semantic distance using the "learning and inference with schemas and analogies" (LISA) model. Our findings indicated that even mild increases in stress impair analogical reasoning. However, the decrement does not seem to directly involve the integration of relations, but rather is due to a shift in decision strategy: Under stress, people show an increased tendency to endorse analogies as valid when the terms in the individual pairs are semantically
\end{abstract}

M. Vendetti $(\bowtie) \cdot$ B. J. Knowlton · K. J. Holyoak Department of Psychology, University of California, 405 Hilgard Avenue,

Los Angeles, CA 90095-1563, USA

e-mail: michael.vendetti@ucla.edu related to each other, even if the overall analogical relationship is not valid.

Keywords Anxiety · Decision-making $\cdot$ Computational model $\cdot$ Working memory $\cdot$ Prefrontal cortex

\section{Stress and cognitive processes}

In real-world situations, complex reasoning must often be performed under conditions that evoke acute stress. Acute stress has been shown to negatively impact a variety of cognitive processes, including memory (Ishizuka, Hellier, \& Beversdorf, 2007; Koessler, Engler, \& Riether, 2009), decision-making (Preston, Stansfield, \& Buchanan, 2007; Lighthall, Mather, \& Gorlick, 2008; Porcelli \& Delgado, 2009; Starcke, Wolf, \& Markowitsch, 2008), mental arithmetic (Beilock \& Carr, 2005), and fluid reasoning (Gimmig, Huguet, \& Caverni, 2006).

The larger literature on the relationship between induced stress and working memory performance (Arnsten, 2000; Arnsten \& Goldman-Rakic, 1998; Beilock \& Carr, 2005; Bishop, 2007; Derekshan \& Eysenck, 2009; Fales et al., 2008; Gimmig et al., 2006; Kern, Oakes, \& Stone, 2008) is consistent with the interpretation that stress impairs function of the prefrontal cortex (PFC).

One type of cognitive process that has not been extensively investigated with respect to induced stress is analogical reasoning. Analogical reasoning allows comparisons between featurally dissimilar situations to be performed on the basis of structural relationships (for recent reviews, see Gentner, 2010; Halford, Wilson, \& Phillips, 2010; Holyoak, 2012). Analogical reasoning plays an important role in many cognitive tasks, including problem solving (Gick \& Holyoak, 1980), developing scientific theories (Dunbar \& 
Klahr, 2012; Holyoak \& Thagard, 1995), guiding consumer decisions (Markman \& Loewenstein, 2010), and teaching mathematics (Richland, Zur, \& Holyoak, 2007). It is therefore an important goal to investigate the mechanisms of analogical reasoning, including the possible impact of factors such as stress.

\section{Reasoning with verbal analogies}

A classic type of problem that can be used to assess analogical reasoning is proportional verbal analogies of the form A:B :: C:D (e.g., FATHER:SON :: INVENTOR: INVENTION). In these four-term analogy problems, the reasoner must extract the relationship between the pair of concepts that comprise $\mathrm{A}: \mathrm{B}$ and then evaluate whether a corresponding relation holds between the C:D pair. For example, for the pair FATHER:SON, one salient relationship is that FATHER produces a SON. The "produce" relationship allows a systematic mapping to be established between FATHER and INVENTOR (both producers) and between SON and INVENTION (both products), indicating that the analogy is valid. Importantly, this type of "semantically distant" analogy is more difficult to solve (as measured by either errors or response times) than a problem in which the concept pairs are more similar (e.g., FATHER:SON :: MOTHER:DAUGHTER; Green, Kraemer, \& Fugelsang, 2010, 2012).

Verbal analogy problems have a long history as psychometric measures of individual differences in analogical reasoning ability (Spearman, 1923, 1946). They have also figured prominently in both behavioral studies of the sources of difficulty in analogical reasoning (Green, Fugelsang, \& Dunbar, 2006a; Morrison et al., 2004; Sternberg, 1977; Sternberg \& Nigro, 1980) and in neuroimaging studies aiming to identify the neural substrates of such reasoning (Bunge, Helskog, \& Wendelken, 2009; Bunge \& Wendelken, 2009; Bunge, Wendelken, \& Badre, 2005; Green et al., 2010, 2012; Krawczyk, McClelland, \& Donovan, 2011). A general conclusion from the neuroimaging work has been that extracting and comparing relations between dissimilar pairs of objects (as in the above FATHER:SON :: INVENTOR:INVENTION example) selectively activates the left rostrolateral PFC (for a review, see Knowlton \& Holyoak, 2009).

Using a task that required identifying corresponding objects in two visual scenes presented simultaneously, Tohill and Holyoak (2000) found that stress (induced by performing a prior difficult arithmetic task) led to a shift from mapping on the basis of a common relation (e.g., choosing a tree as the match to a boy because both restrained a dog) to mapping on the basis of a more direct featural overlap (e.g., choosing a man as the match to the boy). This decrement in analogical mapping due to induced stress occurred even when participants were given a practice example showing that the relational map was the correct response. The negative impact of stress on relation-based mapping might reflect reduced working memory capacity under stress and/or a reduction in the ability to control interference from featural distractors (Cho, Holyoak, \& Cannon, 2007; Cho et al., 2010). Importantly, in Tohill and Holyoak's study, participants did not receive any feedback indicating that featural mappings were incorrect. It is therefore possible that those in the stressed condition may simply have elected to use the simpler strategy of reporting featural rather than relational matches.

To the extent that the process of integrating relations in analogical reasoning depends on PFC function, induced stress may affect one's ability to integrate relations, perhaps inducing a shift to a heuristic strategy based more on semantic similarity. For example, an invalid verbal analogy such as FATHER:SON :: NEPHEW:COUSIN may be a "tempting lure," especially under stress, because the salient pairwise relations (FATHER is a relative of SON, NEPHEW is a relative of COUSIN) support a positive decision. Thus, stress may increase the rate of false alarms to invalid analogies that involve salient semantic relationships within the $\mathrm{A}: \mathrm{B}$ and $\mathrm{C}: \mathrm{D}$ pairs.

In the present study, we investigated how stress impacts analogical reasoning with four-term verbal analogy problems. We used materials developed by Green et al. (2010; see also Green et al., 2012), in which the semantic distance of the $C: D$ pair relative to the A:B pair was varied. Green et al. found that as semantic distance increased (e.g., FATHER: SON :: MOTHER:DAUGHTER vs. FATHER:SON :: INVENTOR:INVENTION), accuracy declined and solution times increased. These behavioral changes were accompanied by an increase in BOLD activity within the left frontopolar cortex, an area that is typically associated with relational integration (Bunge et al., 2005; Green, Fugelsang, Kraemer, et al., 2006b).

By varying semantic distance within analogy problems, with or without induced stress, we sought to discriminate between alternative hypotheses concerning the mechanism by which stress may impact analogical reasoning. If stress impairs the capacity to perform relational integration (perhaps by reducing the processing efficiency of the rostrolateral PFC), we would expect to observe greater performance deficits under stress for problems involving high semantic distance. Alternatively, stress may trigger a more global strategy shift toward basing positive responses on the detection of individual semantic relationships between concepts. Given that the foils always involved closely related concepts within both $A: B$ and $C: D$ pairs, such a strategy shift would be expected to lead to a general increase in false alarms, independent of the semantic distance between the $A: B$ and $C: D$ pairs. 


\section{Method}

\section{Participants}

A group of 58 undergraduates (48 female, 10 male) at the University of California, Los Angeles, participated for partial credit toward the requirements of an undergraduate psychology class. Informed written consent was obtained for each participant according to the ethical guidelines established by the UCLA Office of Human Research Protection Program.

Design, materials, and procedure

The design of the study was a 2 (group: to-be-stressed vs. no-stress control) $\times 2$ (time: before stress induction vs. control/after stress induction) $\times 2$ (semantic distance: near vs. far analogy trials) $\times 2$ (validity: valid vs. invalid analogy trials). The induced-stress manipulation was a betweensubjects variable; the other three factors were manipulated within subjects.

The verbal analogy problems were modified versions of those used by Green et al. (2010). There were a total of 120 analogy problems, each consisting of four words in an $A: B$ :: C:D format. Following Green et al. (2010), problems were subdivided into four types: valid near (40 problems), valid far (40), invalid near (20), and invalid far (20). Valid trials were those for which the A:B relationship was proportional to that of the C:D pair. Near trials were those for which the C:D concepts were drawn from a category semantically similar to that of the A:B pair. For example, if the A:B term was NOSE: SCENT, a near C:D term could be TONGUE:TASTE. Far trials were those for which the $\mathrm{C}: \mathrm{D}$ concepts were drawn from a different semantic category than the A:B terms (e.g., NOSE: SCENT :: ANTENNA:SIGNAL). (For more detailed information about the stimuli, see Green et al., 2010.)

Participants were tested individually. During the informed consent process, participants read that the experiment would be measuring how performance on analogy problems relates to mental arithmetic ability. Participants were informed that in addition to solving analogy problems, they would also be performing a mental arithmetic task. Participants then were asked to fill out the State-Trait Anxiety Inventory (STAI; Spielberger, Gorsuch, \& Lushene, 1970). After this, all participants were brought into the testing room, which consisted of a desk, two chairs, and a computer that controlled the presentation of analogy problems. The computer was a Mac Mini Intel, using Mac OS 10.5 and connected to a 17-in. CRT monitor, and the experiment was presented using Superlab (Cedrus Corp., 2004). After having read the instructions, participants went through three analogy practice trials with feedback along with the experimenter.
Each trial began with a brief fixation cross, followed by presentation of the $A: B$ and $C: D$ pairs, with the $A: B$ pair appearing on the monitor above the C:D pair. Participants were instructed to press a key with their right index finger if the analogy was valid and to hit a different key with their left index finger if the analogy was invalid. They were instructed to solve each trial as quickly and accurately as possible. Feedback was provided under two conditions: if either the participant made the wrong decision or $8 \mathrm{~s}$ had elapsed before a decision was made. If the participant's choice was wrong, the screen displayed "Wrong!"; if the participant took too long, the screen displayed "Too Slow!" Both types of feedback were shown centered in red font for $1 \mathrm{~s}$, after which the next trial began. There were no differences in the amounts of feedback between the two groups for either Block $1[F(1,55)<1, p>.10]$ or Block $2[F(1,55)=1.4$, $p>.10]$.

Two blocks of trials were administered. Each block contained a 2:1 ratio of valid to invalid trials. The proportions of near and far problems were equal within each block. The first block comprised 39 analogy trials. Neither group received any special instructions prior to Block 1 . Following completion of Block 1, the participants were asked to perform a mental arithmetic task. For participants who were assigned to the to-be stressed group, the mental arithmetic task was actually a modified version taken from the Trier Social Stress Test (TSST; Hopko, Ashcraft, \& Gute, 1998; Kellogg, Hopko, \& Ashcraft, 1999; Kirschbaum, Pirke, \& Hellhammer, 1993). Specifically, their task was to count backward, out loud, starting from 1,000 and subtracting 13 (" $1,000,987$," etc.). During participants' attempts to perform this mental arithmetic task, every $10 \mathrm{~s}$ they were told by the experimenter to go faster. Each time an error was made, the experimenter instructed the participant to start over. Participants in the stressed condition were unaware of how long they would have to complete this task, but all participants were told to stop after $1 \mathrm{~min}$. They were then warned that they would have to perform a similar mental arithmetic task later on in the experimental session. This warning was included to induce a stronger sense of worry about their performance. (The arithmetic task was not in fact repeated.) In contrast, participants assigned to the control condition (no stress) were simply asked to count forward by one starting from zero for the same amount of time, with no interruption by the experimenter or expectation of an additional arithmetic test later.

Immediately after the mental arithmetic task, participants in both groups were asked to fill out the State form of the STAI (i.e., a second assessment of stress was obtained). They then completed the second block of analogy problems. Block 2 comprised 78 trials of the same types of problems as had been administered in Block 1. After finishing Block 2, all participants completed 
the State form of the STAI for a third time. Following this, they were thanked for their participation in the study and fully debriefed.

\section{Results}

Trait and state anxiety scores

The mean trait and state scores for the two conditions are shown in Fig. 1. Trait anxiety scores were collected at the beginning of the study and provided a check that the participants randomly assigned to the two conditions had similar levels of trait anxiety as measured by the STAI (Spielberger et al., 1970). The conditions did not differ significantly in trait anxiety scores: control $(n=28, M=36.93)$, to-be-stressed $(n=29, M=35.21), t(55)=0.79, p=.435$. The trait anxiety scores for both groups were well within the normal range for young adults (norm $M=35.85$; Spielberger et al., 1970).

As described above, state anxiety scores were collected at three points during the study: (1) immediately before administration of the trait anxiety subscale, (2) immediately after the mental arithmetic task that followed completion of Block 1, and (3) after completing Block 2. A 2 (control vs. to-bestressed) $\times 3$ (time of state anxiety test) mixed-subjects ANOVA yielded a significant interaction, $F(2,112)=9.21$, $M S E=58.95, p<.001$. Follow-up independent $t$ tests for each state assessment time point yielded a significant difference between the groups only at the second assessment time, immediately after completing the mental arithmetic task, $t(55)=$ $4.43, p<.001$. These results confirm that the stress induction procedure succeeded in increasing state anxiety scores for the to-be-stressed group, with the level returning to baseline by the conclusion of Block 2.

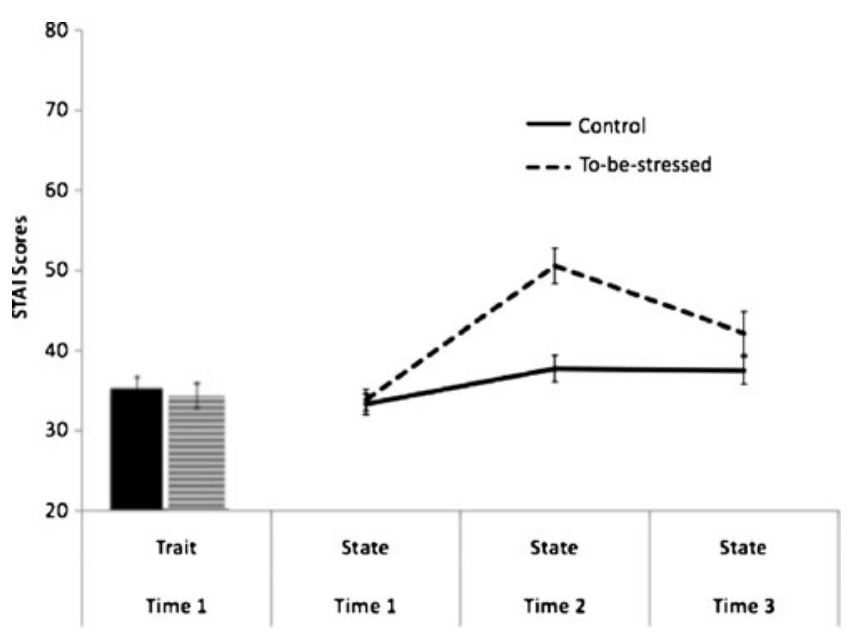

Fig. 1 State and trait anxiety scores for the control and to-be-stressed conditions. Participants in the latter condition received stress induction prior to the state evaluation at Time 2. Error bars indicate $\pm 1 S E M$
Performance on analogy task

We will report analyses based on accuracy, as response times did not reveal any influence of the stress manipulation. The main analyses were performed on $d^{\prime}$ scores, calculated separately for the near and far problem types. In calculating $d^{\prime}$ for individual participants, scores at one of the extremes $(0$ or 1$)$ were replaced by $0.5 / n$ or $(n-0.5) / n$, respectively, where $n=$ number of trials (Stanislaw \& Todorov, 1999). The results are shown in Fig. 2. We performed a 2 (Block 1 vs. Block 2$) \times 2$ (semantic distance $) \times 2$ (group) ANOVA on the $d^{\prime}$ scores. As is shown in Fig. 2, we found a main effect of block, such that $d^{\prime}$ was significantly greater in Block $1(M=3.32)$ than in Block $2(M=2.90), F$ $(1,56)=6.16, M S E=1.67, p=.016$. A main effect of semantic distance was also obtained, with $d^{\prime}$ significantly greater for near problems $(M=3.68)$ than for far problems $(M=2.55), F(1,56)=35.47, M S E=2.12, p<.001$. Most importantly, a reliable Block $\times$ Group interaction was obtained, $F(1,56)=5.54, M S E=1.668, p=.022$. Followup $t$ tests revealed no reliable differences in $d^{\prime}$ scores between the two groups in Block 1 (control, $M=3.29$; to-bestressed, $M=3.36), t(55)=0.17, p=.868$. However, the two groups did differ reliably on Block 2 , after the stress manipulation (control, $M=3.27$; stressed, $M=2.54$ ), $t(55)=2.29$, $p=.027$. The three-way interaction among block, group, and semantic distance was not reliable, $p>.05$.

In addition to calculating $d^{\prime}$ scores on the basis of signal detection theory, we also calculated the corresponding bias scores. If the difference in accuracy between the two conditions in Block 2 were solely due to a change in bias, we would expect the stressed condition to yield a greater overall proportion of "yes" responses (i.e., an increase in hits as well in false alarms). However, the two groups did not differ reliably in an ANOVA based on bias scores for Block 2, $F$ $(1,56)=1.08, p>.10$.

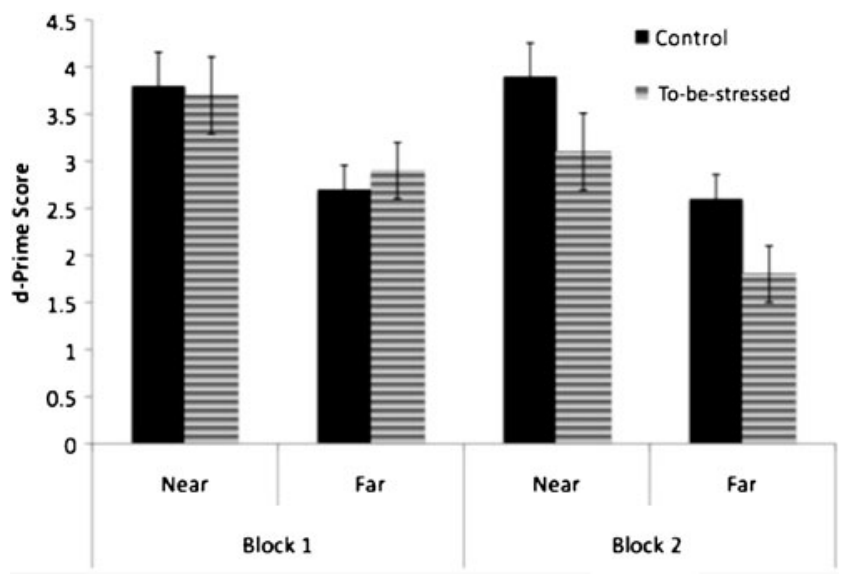

Fig. 2 Overall $d$ 'scores for accuracy as a function of semantic distance (near vs. far) and condition. Participants in the to-be-stressed condition received stress induction prior to Block 2. Error bars indicate $\pm 1 S E M$ 
As is shown in Fig. 3, the difference in $d$ 's between the two groups across blocks was in fact mainly due to an increase in false alarms across both levels of semantic distance for participants in the stressed group as compared to those in the control group. (The proportions of hits on valid trials are not reliably different between groups.) We ran a multiple regression analysis on false alarms produced in Block 2 using the false alarm rate in Block 1 as one predictor, with the change in participant state anxiety scores between Time Points 1 and 2 as an additional predictor. This analysis revealed that, controlling for initial false alarm rates in Block 1, a positive relationship still remained between changes in state anxiety scores and false alarms in Block 2, $F(1,53)=17.12, M S E=0.011, p<.001$.

\section{LISA simulation of verbal analogy performance}

We modeled our results using the "learning and inference with schemas and analogies" (LISA) model (Hummel \& Holyoak, 1997, 2003), a symbolic-connectionist model of analogical mapping. LISA uses a hierarchical structure to represent objects and to predicate relations within each pair of the analogy. The lowest level contains semantic units that represent features of each of the concepts within an analogue [for our simulations, simple propositions such as produce (father, son)], with separate pools of units for objects (e.g., father, son) and for roles (e.g., producer, produced). For example, the objects father and son might be coded by distributed patterns across semantic units such as [human, adult, child, male], while the roles producer and produced might be coded by patterns across semantic units such as [action, generative]. The upper levels of the hierarchy comprise various types of structure units that represent

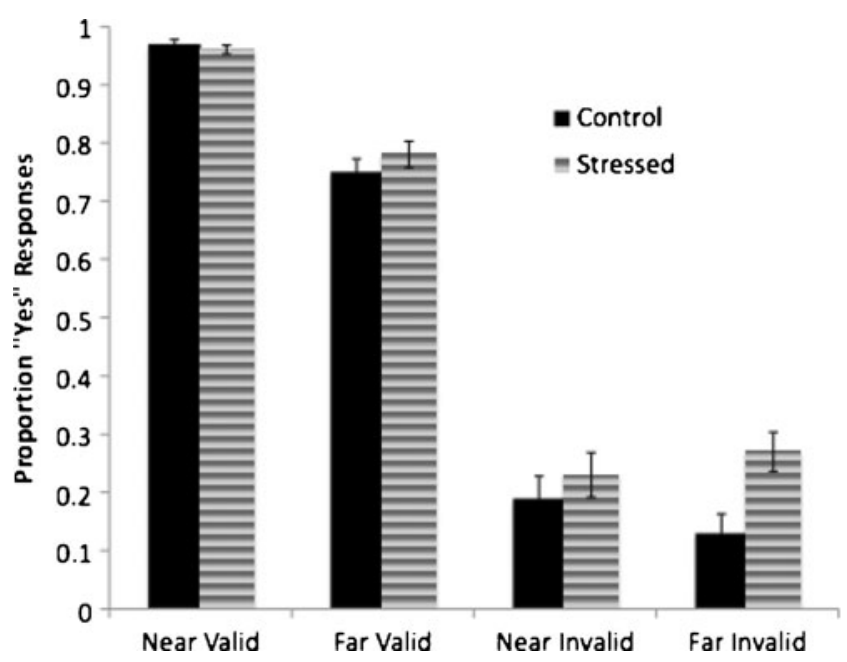

Fig. 3 Proportions of "yes" responses (i.e., hits for valid analogy trials and false alarms for invalid trials), by semantic distance (near vs. far) and condition, after the stress manipulation (i.e., in Block 2). Error bars indicate $\pm 1 S E M$ the components of propositions. These include units for objects and roles, as well as role-binding (RB) units that link objects to the appropriate roles (e.g., father + producer, son + produced). Finally, the highest level is a proposition (P) unit that binds multiple RB units into a complete proposition.

As sketched in Fig. 4, we modeled the verbal analogy task by coding the A:B and C:D pairs as two separate analogues and then mapping the former (in the terms of the LISA model, the driver) to the latter (the recipient). The model operates by updating mapping connections (not illustrated) that capture the correspondences between the components of the two analogues (i.e., forming connections between the objects $\mathrm{A}$ and $\mathrm{C}$ and between $\mathrm{B}$ and $\mathrm{D}$, as well as between the corresponding role and RB units). As a simple index of the overall "goodness" of the mapping between the analogues, we used a similarity score developed for LISA by Taylor and Hummel (2010, p. 232, Eq. 5) that is sensitive to both the relational and featural overlap between analogues (the "total similarity score"). We made the assumption that the probability of responding "yes" to an analogy problem will increase with LISA's similarity score. (For more detail on the operation of LISA, see Hummel \& Holyoak, 1997, 2003; Taylor \& Hummel, 2009.)

Our aim was to model the three major effects identified in the behavioral data summarized in Fig. 3: (1) For both conditions, the proportion of "yes" responses was greater for valid than for invalid problems; (2) for both conditions, the proportion of "yes" responses was greater for near than for far problems; and (3) the stressed group gave more "yes" responses for invalid problems than did the control condition. In order to evaluate the LISA model's sensitivity to the first two factors, we created four simulations: near valid, far valid, near invalid, and far invalid analogies, representing the four basic problem types. For all simulations, the A and $\mathrm{B}$ (and C and D) concepts were created so as to have $50 \%$ overlap across semantic units, reflecting the close semantic similarity between the concepts within each pair. Valid trials were constructed by including identical predicates in the source and target analogues (e.g., if produces was the predicate for FATHER:SON, produces was also the predicate for INVENTOR:INVENTION). Invalid trials, by their very nature, required predicates that differed. Near problems were created with more overlap of semantic units among the objects and roles comprising the A:B and C:D pairs, as compared to the far problems (approximately $60 \%$ and $45 \%$ overlap, respectively).

We first applied the LISA model to the control condition to see whether it could account for the basic effects of validity and semantic distance. All of the parameters were fixed at their default values (see Hummel \& Holyoak, 2003, Appx. A), and the total similarity score (Taylor \& Hummel, 2010) was calculated. Figure 5 shows the proportions of 
Fig. 4 Modeling the verbal analogy task in the LISA model. Each proposition within the analogy consists of a set of hierarchical units used to represent the concepts in each pair as well as their relationships

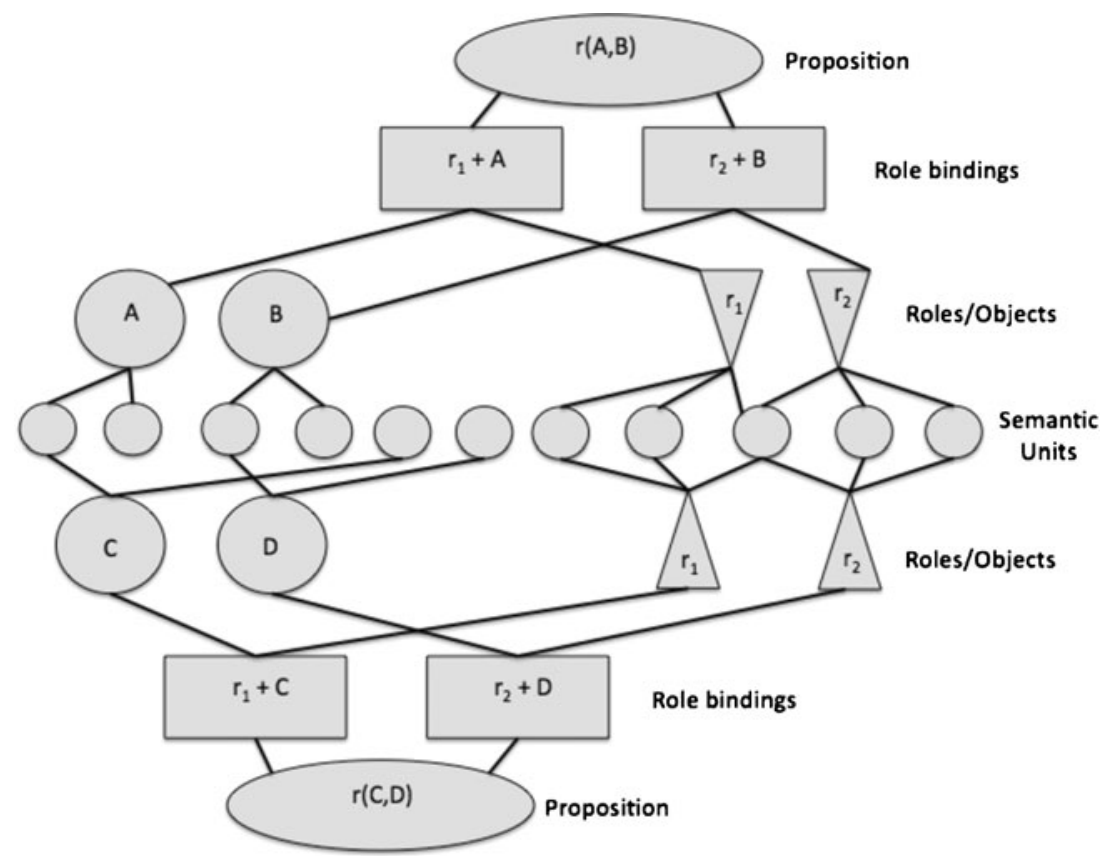

"yes" responses across the four problem types for the control condition along with LISA's predictions (based on a linear rescaling of the similarity scores to fit the human data). The model was able to account for $98 \%$ of the variance among the four conditions, thus capturing both the basic effect of validity and also the semantic distance effect (Green et al., 2010).

We next explored possible approaches to simulating the impact of stress on the proportions of "yes" responses (see

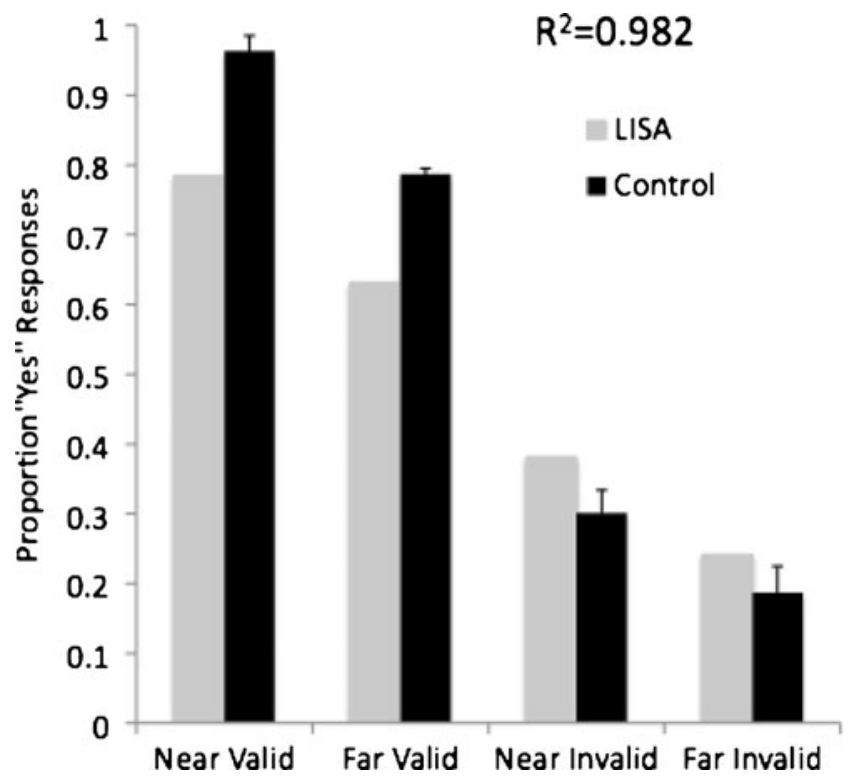

Fig. 5 Proportions of "yes" responses (i.e., hits for valid trials and false alarms for invalid trials) as a function of semantic distance (near vs. far) for the control (no-stress) condition in Block 2, along with predictions derived from the LISA model (see the text) for more details. Error bars indicate $\pm 1 S E M$
Fig. 3). In previous simulation work, LISA has been used to model the impact of frontal damage, by varying its parameters for inhibition and for learning mapping connections (Morrison et al., 2004); to model the impact of normal aging, by varying its inhibition parameter (Viskontas, Morrison, \& Holyoak, 2004); and to model developmental changes, also by varying the inhibition parameter (Morrison, Doumas, \& Richland, 2011). However, in all of these previous simulations, a key qualitative finding was a change in the ability to solve problems at higher levels of relational complexity. In the present study, the semantic distance factor can be viewed as a type of complexity manipulation, as supported by the fact that higher semantic distance is known to increase the activation of rostrolateral PFC (Green et al., 2010, 2012). However, we found that stress did not selectively impair performance with far problems. Rather, the empirical finding was that stress increased the false alarm rate for invalid problems at both levels of semantic distance, while having a negligible impact on hit rates (see Fig. 3).

Not surprisingly, given the fact that stress did not interact with semantic distance in the present experiment, we found that its impact could not be captured by manipulating LISA's inhibition or learning rate parameters, as both types of changes inherently yield such an interaction. These negative findings based on the LISA model imply that the impact of stress is not to directly impair relational processing, but rather to trigger a general shift in decision strategy. Specifically, it seemed plausible that under stress, participants might sometimes respond "yes" simply because the concepts within each pair (A:B and also C:D) are closely related. To model this possibility, we tested a simple model that predicts how the choice between two alternative strategies for solving analogy problems 
(responding based on how proportional the relations between the $A: B$ and $C: D$ pairs are vs. responding on the basis of semantic similarity within each pair) may be influenced by the impact of stress. Specifically, we assumed that with probability $p$ (estimated by the LISA prediction for the control condition), stressed participants respond on the basis of analogical mapping, and with probability $1-p$, they simply respond "yes" due to the featural similarity within each pair of concepts [i.e., probability of "yes" response under stress $=p$ (LISA prediction for control) $+(1-p)]$.

A regression analysis revealed that this model accounted for $95 \%$ of the variance in "yes" responses across the four conditions for the to-be-stressed condition (with the value of $p$ equal to .90). Thus, the most successful computational account of the impact of acute stress on analogy performance in our experiment was that, on a small proportion of trials, stress induces a shift to a nonanalogical decision strategy.

\section{Discussion}

In the present study, we found that mild induced stress had a different influence on analogical reasoning than did a variation in the complexity of verbal analogy problems (the semantic distance between A:B and C:D pairs). In accord with previous findings (Green et al., 2010, 2012), increasing semantic distance decreased hits and increased false alarms. In contrast, inducing stress led to an increase in false alarms without decreasing hits. Moreover, no interaction was observed between the influences of semantic distance and stress on solution accuracy. This pattern suggests that stress did not impair the capacity to integrate relations, which would presumably be more difficult in the far semantic condition. Rather, the present findings indicate that stress has its impact at the level of the decision process, encouraging a strategy of making positive responses based on the fact that the individual word pairs are semantically related. From the neuroimaging literature concerning analogical reasoning (Bunge et al., 2005; Green et al., 2010, 2012), one would suspect that to the extent that stress induction reduces the likelihood of relational integration, less activation in rostrolateral PFC areas would be observed for both near and far analogies relative to control conditions.

The LISA model (Hummel \& Holyoak, 1997, 2003), a symbolic-connectionist model of analogical mapping, was able to capture the semantic distance effect. By manipulating the amount of semantic overlap between the A:B and C: $\mathrm{D}$ terms in LISA representations, a similarity measure derived from LISA (Taylor \& Hummel, 2010) was able to explain $98 \%$ of the variance in analogy selections by participants in the control condition. The changes in selections that accompanied stress could be accounted for by a model in which, on a small proportion of trials, stressed participants were assumed to give a positive response regardless of the actual validity of the analogy problem.

These modeling results have implications for understanding how stress impacts the ability to solve verbal analogies. Under stress, a reasoner is more likely to use a simpler decision strategy, endorsing an invalid analogy due to the semantic similarity between the items in each of its individual word pairs. In view of the present findings, we hypothesize that participants in the stressed condition of Tohill and Holyoak (2000) may have been capable of performing relational mapping, but instead, more often opted for a strategy of identifying object correspondences on the basis of featural similarity.

An important direction for future research will be to examine individual differences in the effects of stress on analogical reasoning. For example, previous findings have shown that individuals who score higher on working memory measures suffer more on math and inductive reasoning problems when under stress (Beilock \& Carr, 2005; Gimmig et al., 2006). It may be that inducing stress would have a greater impact on analogical reasoning for those individuals with greater working memory span. Our results raise the possibility that, while high-span individuals may generally perform better on analogical reasoning tasks, they may also have a greater tendency under stress to shift to a heuristic strategy based on the similarity within pairs.

In conclusion, the present study provides evidence concerning how induced stress may impact the ability to reason using analogies. Specifically, we found that induced stress led to a selective increase in false alarms in evaluating invalid analogy problems, apparently due to a tendency to use a simpler decision strategy. Unlike a number of other factors known to impair analogical reasoning, such as prefrontal damage (Morrison et al., 2004) and normal aging (Viskontas et al., 2004), mild induced stress may not necessarily impair one's ability to integrate relations. Whereas these other factors clearly interact with the complexity of analogy problems, the impact of stress proved to be constant across simpler problems (near semantic distance) and more complex ones (far semantic distance). If mild stress encourages a general shift to a simpler decision strategy, it would seem that its impact could be overcome by interventions that encourage continued focus on relational processing. The present findings thus may have important implications for developing methods to help cope with moderate levels of stress, of the sort that often occur in educational environments during examinations. Of course, the impact of stress may be qualitatively different at more extreme levels, such as those associated with anxiety disorders, depression, and posttraumatic stress syndrome, or with analogies that are substantially more complex than those used here. 
Author note This research was supported by ONR Grant N000140810186. We thank John Hummel for assistance with the LISA simulations and Branden Bio, Matthew Rosenberg, and Howard Yang for assistance with data collection.

\section{References}

Arnsten, A. F. (2000). Stress impairs prefrontal cortical function in rats and monkeys: Role of dopamine D1 and norepinephrine alpha-1 receptor mechanisms. Progress in Brain Research, 126, 183-192.

Arnsten, A. F., \& Goldman-Rakic, P. S. (1998). Noise stress impairs prefrontal cortical cognitive function in monkeys: Evidence for a hyperdopaminergic mechanism. Archives of General Psychiatry, $55,362-368$.

Beilock, S. L., \& Carr, T. H. (2005). When high-powered people fail: Working memory and "choking under pressure" in math. Psychological Science, 16, 101-105. doi:10.1111/j.09567976.2005.00789.x

Bishop, S. J. (2007). Neurocognitive mechanisms of anxiety: An integrative account. Trends in Cognitive Sciences, 11, 307-316. doi:10.1016/j.tics.2007.05.008

Bunge, S. A., Helskog, E. H., \& Wendelken, C. (2009). Left, but not right, rostrolateral prefrontal cortex meets a stringent test of the relational integration hypothesis. NeuroImage, 46, 338-342. doi:10.1016/j.neuroimage.2009.01.064

Bunge, S. A., \& Wendelken, C. (2009). Comparing the bird in the hand with the ones in the bush. Neuron, 62, 609-611. doi:10.1016/j. neuron.2009.05.020

Bunge, S. A., Wendelken, C., Badre, D., \& Wagner, A. D. (2005). Analogical reasoning and prefrontal cortex: Evidence for separable retrieval and integration mechanisms. Cerebral Cortex, 15, 239-249. doi:10.1093/cercor/bhh126

Cedrus Corp. (2004). Superlab 4.0 [Computer software]. San Pedro, CA: Author.

Cho, S., Holyoak, K. J., \& Cannon, T. D. (2007). Analogical reasoning in working memory: Resources shared among relational integration, interference resolution, and maintenance. Memory and Cognition, 35, 1445-1455. doi:10.3758/BF03193614

Cho, S., Moody, T. D., Fernandino, L., Mumford, J. A., Poldrack, R. A., Cannon, T. D., . . . Holyoak, K. J. (2010). Common and dissociable prefrontal loci associated with component mechanisms of analogical reasoning. Cerebral Cortex, 20, 524-533. doi:10.1093/cercor/bhp121

Derakshan, N., \& Eysenck, M. W. (2009). Anxiety, processing efficiency, and cognitive performance: New developments from attentional control theory. European Psychologist, 14, 168-176.

Dunbar, K. N., \& Klahr, D. (2012). Scientific thinking and reasoning. In K. J. Holyoak \& R. G. Morrison (Eds.), The Oxford handbook of thinking and reasoning (pp. 701-718). New York, NY: Oxford University Press.

Fales, C. L., Barch, D. M., Burgess, G. C., Schaefer, A., Mennin, D. S., Gray, J. R., . . . Braver, T. S. (2008). Anxiety and cognitive efficiency: Differential modulation of transient and sustained neural activity during a working memory task. Cognitive, Affective, \& Behavioral Neuroscience, 8, 239-253. doi:10.3758/ CABN.8.3.239

Gentner, D. (2010). Bootstrapping the mind: Analogical processes and symbol systems. Cognitive Science, 34, 752-775. doi:10.1111/ j.1551-6709.2010.01114.x

Gick, M. L., \& Holyoak, K. J. (1980). Analogical problem solving. Cognitive Psychology, 12, 306-355.

Gimmig, D., Huguet, P., Caverni, J.-P., \& Cury, F. (2006). Choking under pressure and working memory capacity: When performance pressure reduces fluid intelligence. Psychonomic Bulletin \& Review, 13, 1005-1010. doi:10.3758/BF03213916

Green, A. E., Fugelsang, J. A., \& Dunbar, K. N. (2006a). Automatic activation of categorical and abstract analogical relations in analogical reasoning. Memory and Cognition, 34, 1414-1421. doi:10.3758/BF03195906

Green, A. E., Fugelsang, J. A., Kraemer, D. J. M., Shamosh, N. A., \& Dunbar, K. N. (2006b). Frontopolar cortex mediates abstract integration in analogy. Brain Research, 1096, 125-137.

Green, A. E., Kraemer, D. J. M., Fugelsang, J. A., Gray, J. R., \& Dunbar, K. N. (2010). Connecting long distance: Semantic distance in analogical reasoning modulates frontopolar cortex activity. Cerebral Cortex, 20, 70-76. doi:10.1093/cercor/bhp081

Green, A. E., Kraemer, D. J. M., Fugelsang, J. A., Gray, J. R., \& Dunbar, K. N. (2012). Neural correlates of creativity in analogical reasoning. Journal of Experimental Psychology: Learning, Memory, and Cognition, 38, 264-272. doi:10.1037/a0025764

Halford, G. S., Wilson, W. H., \& Phillips, S. (2010). Relational knowledge: The foundation of higher cognition. Trends in Cognitive Sciences, 14, 497-505.

Holyoak, K. J. (2012). Analogy and relational reasoning. In K. J. Holyoak \& R. G. Morrison (Eds.), The Oxford handbook of thinking and reasoning (pp. 234-259). New York: Oxford University Press.

Holyoak, K. J., \& Thagard, P. (1995). Mental leaps: Analogy in creative thought. Cambridge: MIT Press.

Hopko, D. R., Ashcraft, M. H., Gute, J., Ruggiero, K. J., \& Lewis, C. (1998). Mathematics anxiety and working memory: Support for the existence of a deficient inhibition mechanism. Journal of Anxiety Disorders, 12, 343-355.

Hummel, J. E., \& Holyoak, K. J. (1997). Distributed representations of structure: A theory of analogical access and mapping. Psychological Review, 104, 427-466. doi:10.1037/0033-295X.104.3.427

Hummel, J. E., \& Holyoak, K. J. (2003). A symbolic-connectionist theory of relational inference and generalization. Psychological Review, 110, 220-264. doi:10.1037/0033-295X.110.2.220

Ishizuka, K., Hillier, A., \& Beversdorf, D. Q. (2007). Effect of the cold pressor test on memory and cognitive flexibility. Neurocase, 13, $154-157$.

Kellogg, J. S., Hopko, D. R., \& Ashcraft, M. H. (1999). The effects of time pressure on arithmetic performance. Journal of Anxiety Disorders, 13, 591-600.

Kern, S., Oakes, T. R., Stone, C. K., McAuliff, E. M., Kirschbaum, C., \& Davidson, R. J. (2008). Glucose metabolic changes in the prefrontal cortex are associated with HPA axis response to a psychosocial stressor. Psychoneuroendocrinology, 33, 517-529. doi:10.1016/j.psyneuen.2008.01.010

Kirschbaum, C., Pirke, K. M., \& Hellhammer, D. H. (1993). The "Trier Social Stress Test" - A tool for investigating psychobiological stress responses in a laboratory setting. Neuropsychobiology, 28, $76-81$.

Knowlton, B. J., \& Holyoak, K. J. (2009). Prefrontal substrate of human relational reasoning. In M. S. Gazzaniga (Ed.), The cognitive neurosciences (4th ed., pp. 1005-1017). Cambridge: MIT Press.

Koessler, S., Engler, H., Riether, C., \& Kissler, J. (2009). No retrievalinduced forgetting under stress. Psychological Science, 20, 13561363. doi:10.1111/j.1467-9280.2009.02450.x

Krawczyk, D. C., McClelland, M., \& Donovan, C. M. (2011). A hierarchy for relational reasoning in the prefrontal cortex. Cortex, 47, 588-597. doi:10.1016/j.cortex.2010.04.008

Lighthall, N. R., Mather, M., \& Gorlick, M. A. (2008). Acute stress increases sex differences in risk seeking in the balloon analogue risk task. PLoS One, 4, e6002.

Markman, A. B., \& Loewenstein, J. (2010). Structural comparison and consumer choice. Journal of Consumer Psychology, 20, 126-137. 
Morrison, R. G., Doumas, L. A. A., \& Richland, L. E. (2011). A computational account of children's analogical reasoning: Balancing inhibitory control in working memory and relational representation. Developmental Science, 14, 516-529. doi:10.1111/ j.1467-7687.2010.00999.x

Morrison, R. G., Krawczyk, D. C., Holyoak, K. J., Hummel, J. E., Chow, T. W., Miller, B. L., . . Knowlton, B. J. (2004). A neurocomputational model of analogical reasoning and its breakdown in Frontotemporal Lobar Degeneration. Journal of Cognitive Neuroscience, 16, 260-271.

Porcelli, A. J., \& Delgado, M. R. (2009). Acute stress modulates risk taking in financial decision making. Psychological Science, 20, 278-283.

Preston, S. D., Stansfield, R. B., Buchanan, T. W., \& Bechara, A. (2007). Effects of anticipatory stress on decision making in a gambling task. Behavioral Neuroscience, 121, 257-263.

Richland, L. E., Zur, O., \& Holyoak, K. J. (2007). Cognitive supports for analogies in the mathematics classroom. Science, 316, 11281129. doi:10.1126/science. 1142103

Spearman, C. (1923). The nature of intelligence and the principles of cognition. London, U.K.: Macmillan.

Spearman, C. (1946). Theory of a general factor. British Journal of Psychology, 36, 117-131.
Spielberger, C. D., Gorsuch, R. L., \& Lushene, R. (1970). STAI: Manual for the State-Trait Anxiety Inventory. Palo Alto: Consulting Psychologists Press.

Stanislaw, H., \& Todorov, N. (1999). Calculation of signal detection theory measures. Behavior Research Methods, Instruments, \& Computers, 31, 137-149. doi:10.3758/BF03207704

Starcke, K., Wolf, O. T., Markowitsch, H. J., \& Brand, M. (2008). Anticipatory stress influences decision making under explicit risk conditions. Behavioral Neuroscience, 122, 1352-1360.

Sternberg, R. (1977). Intelligence, information processing, and analogical reasoning. Hillsdale: Erlbaum.

Sternberg, R. J., \& Nigro, G. (1980). Developmental patterns in the solution of verbal analogies. Child Development, 51, 27-38.

Taylor, E. G., \& Hummel, J. E. (2009). Finding similarity in a model of relational reasoning. Cognitive Systems Research. 10(3), 229 239.

Tohill, J. M., \& Holyoak, K. J. (2000). The impact of anxiety on analogical reasoning. Thinking and Reasoning, 6, 27-40.

Viskontas, I. V., Morrison, R. G., Holyoak, K. J., Hummel, J. E., \& Knowlton, B. J. (2004). Relational integration, inhibition and analogical reasoning in older adults. Psychology and Aging, 19, $581-591$. 